\title{
Influence of tip-sample interaction in a time-domain terahertz scattering near field scanning microscope
}

\author{
A. Thoma ${ }^{\text {a) }}$ and T. Dekorsy \\ Department of Physics and Center for Applied Photonics, University of Konstanz, \\ D-78457 Konstanz, Germany
}

\begin{abstract}
Apertureless near field measurements with a metallic tip are performed in the terahertz frequency range. Lateral scans are recorded for different time delays within a terahertz pulse. The forward scattered terahertz signal strongly depends on the time delay. At larger time delays, the tip-sample interaction leads to additional structures in the scan that do not correspond to a change in topography or dielectric function.
\end{abstract}

Electromagnetic radiation in the terahertz frequency range is of particular interest for the investigation of excitations like phonons ${ }^{1}$ and plasmons ${ }^{2}$ in semiconductors and molecular vibrations in biomolecules. ${ }^{3,4}$ However, lateral variations in sample properties on a scale smaller than the diffraction limited spot size $(\lambda=300 \mu \mathrm{m}$ at $1 \mathrm{THz})$ of the terahertz beam are difficult to detect by conventional terahertz time-domain spectroscopy (TDS). In particular, for spatially resolved spectroscopy and imaging of nanostructures, it is highly desirable to overcome the diffraction limitation at terahertz frequencies.

In order to achieve subwavelength resolution, near field scanning techniques like subwavelength apertures were established in the microwave ${ }^{5,6}$ and visible ${ }^{7,8}$ frequency ranges. The achievable resolution in aperture scanning near field optical microscope (SNOM) is mainly determined by the size of the aperture provided the distance between aperture and sample is smaller than the aperture's size. In the terahertz frequency range, several approaches using apertures were reported with detection in the far field ${ }^{9-12}$ and in the near field. ${ }^{13,14} \mathrm{~A}$ resolution of $7 \mu \mathrm{m}$ at a central frequency of $0.5 \mathrm{THz}$ could be obtained by using a $5 \mu \mathrm{m}$ aperture. ${ }^{14} \mathrm{~A}$ dynamic aperture technique was described by Chen et al. ${ }^{15}$ Low transmission and the difficulty in fabrication of small apertures set constraints to the achievable resolution in practice.

For higher resolution, apertureless ${ }^{16}$ (or scattering) SNOM is advantageous, especially at longer wavelengths. ${ }^{17}$ In the terahertz frequency range, several groups have realized scattering based SNOMs. In these approaches, the scattered radiation from the tip was measured in the near field $^{18,19}$ or in the far field. ${ }^{20-23}$

Terahertz near field images obtained with a tip-based SNOM and detection in the far field so far have been acquired by measuring the peak value of the electric field of the terahertz pulse as a function of the lateral position of the sample. With this approach, a lateral resolution of $150 \mathrm{~nm}$ has been obtained. ${ }^{24}$ In this Letter, apertureless near field measurements obtained in the far field are presented, where the sample was scanned at different time delays of the terahertz pulse. The recorded lateral scans are strongly dependent on the time delay and hence provide a detailed insight into the tip-sample interaction.

\footnotetext{
${ }^{a)}$ Electronic mail: arne.thoma@uni-konstanz.de.
}

The setup was a (standard) terahertz TDS setup in reflection geometry with electro-optic detection. Terahertz pulses were generated using a large area microstructured photoconductive emitter. ${ }^{25} \mathrm{~A}$ Ti:sapphire femtosecond laser providing $60 \mathrm{fs}$ pulses at $800 \mathrm{~nm}$ central wavelength and at a repetition rate of $80 \mathrm{MHz}$ was used for excitation with an average power of $450 \mathrm{~mW}$. The emitter bias was modulated with a bipolar square wave voltage with an amplitude of $14 \mathrm{~V}$ at a frequency of $51 \mathrm{kHz}$, which served as reference frequency for lock-in detection. The $p$-polarized terahertz radiation was focused onto the sample underneath a sharp metal tip by two off axis parabolic mirrors with an angle of incidence of $60^{\circ}$. The tip was prepared by electrochemically etching a $250 \mu \mathrm{m}$ tungsten wire, resulting in a tip radius of $\approx 100 \mathrm{~nm}$. The terahertz pulse reflected in forward direction was collected and focused onto a $1.5 \mathrm{~mm}$ thick (110) oriented ZnTe crystal. A time-delayed probe pulse for electro-optic detection was aligned collinear to the terahertz pulse by using a highresistivity Si beam splitter. A balanced detection scheme employing a quarter wave plate and two photodiodes was used; the intensity difference was demodulated in a lock-in amplifier at the emitter bias modulation frequency. The sample consisted of a $100 \times 100 \mu \mathrm{m}^{2}$ sized gold square with a height of $100 \mathrm{~nm}$ deposited on a glass substrate. The sample was scanned by a two axis stage with $5 \mu \mathrm{m}$ step width, i.e., $1 / 60 \lambda$ at $1 \mathrm{THz}$, underneath the stationary tip. The tip was held at a constant height of approximately $1 \mu \mathrm{m}$ during scanning.

Figure 1 shows a terahertz field transient recorded with the tip in the middle, above the gold structure and above the glass substrate, respectively. The field transient consists of a main peak followed by an oscillatory tail. As the setup is not purged, ringing occurs from water vapor absorption. Its bandwidth ranges from 0.2 to $3 \mathrm{THz}$ (see Fourier transform in inset). The terahertz transient with the glass substrate under the tip varies mainly in amplitude, as the temporal shape is determined by the emitter and both substrate and gold film have a flat spectral response in this wavelength range.

When measuring the electric field of the main peak (corresponding to time delay zero in Fig. 1) and scanning the sample under the tip, the reflected field amplitude changes on a lateral scale much smaller than the central wavelength of the terahertz pulse (Fig. 2): the field change when scanning across the right edge changes within one step of $5 \mu \mathrm{m}$ by $80 \%$ of the maximum difference in the field amplitudes on top of the gold and on the substrate. With the tip withdrawn, the measured signal exhibits no sharp variation on the same 


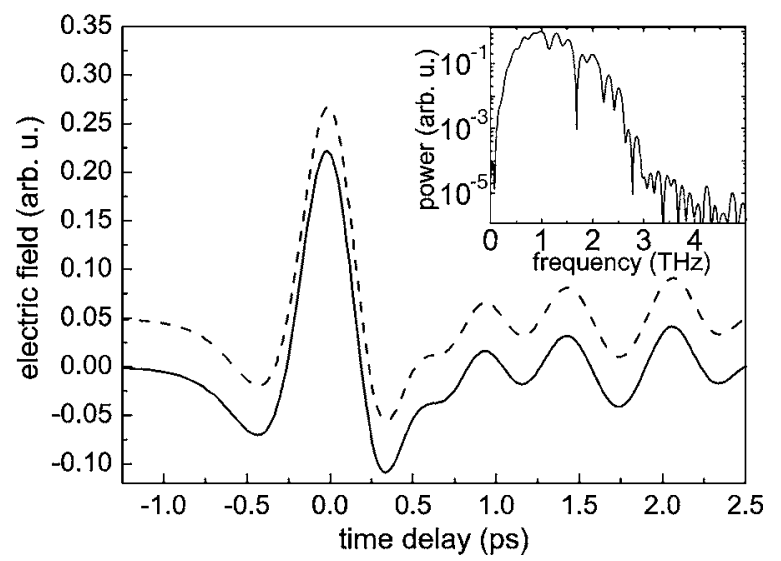

FIG. 1. THz transient reflected from the sample with the tip positioned above the gold structure (solid line) and above the glass substrate (dashed line, offset by 0.05 for clarity). Inset: Normalized Fourier power spectrum of the THz pulse with the tip above the gold structure. Absorption lines are due to water vapor.

length scale. In addition, no structure is observed when $s$-polarized radiation is used since the $s$-polarized terahertz field does not couple effectively to the tip. The increase in the radiation scattered in the forward direction when the tip is above the gold film can be explained by modeling the tip as an antenna. ${ }^{26}$

The signal measured this way clearly shows a one-to-one correspondence to the sample structure with subwavelength resolution. An interesting question is the modulation of the terahertz transient when the lateral scan is recorded at larger time delays $\tau$.

Figure 3 displays several scans at different delays between -0.42 and $+2.06 \mathrm{ps}$ with respect to the main peak. In the lateral scans, the central peaks (where the gold film is underneath the tip) differ in sign and in the absolute value of amplitude (note the different scales in Fig. 3). The sign of the central peak in the lateral scan corresponds to the sign of the electric field in the terahertz transient at the same time delay, i.e., if the terahertz transient displays a maximum or a minimum.

Looking at scans at positive time delays ( $\tau$ greater than zero), additional structures can be seen, which are symmetric around the central peak. At $\tau=0.35 \mathrm{ps}$, the change in electric field is negative above the gold structure. Next to the edges

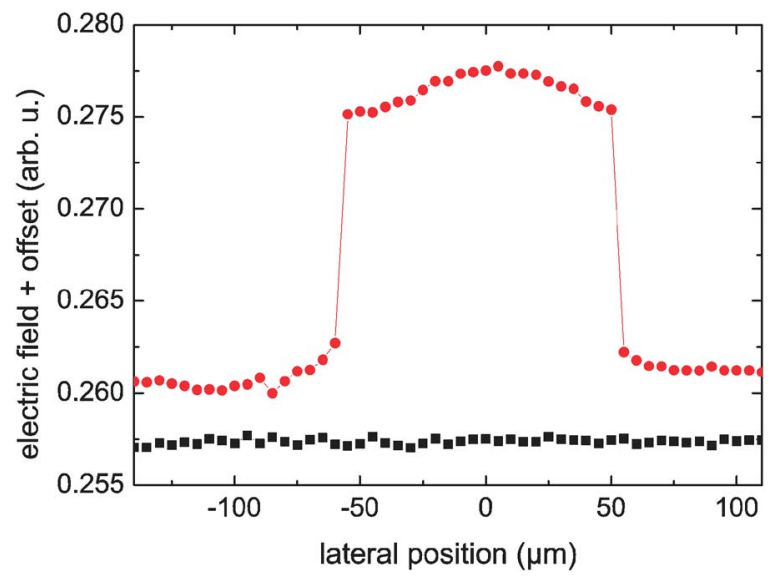

FIG. 2. (Color online) Lateral scan with a step size of $5 \mu \mathrm{m}$ across the gold structure with (red circles) and without a tip (black squares), respectively. The curves are offset with respect to each other for clarity.

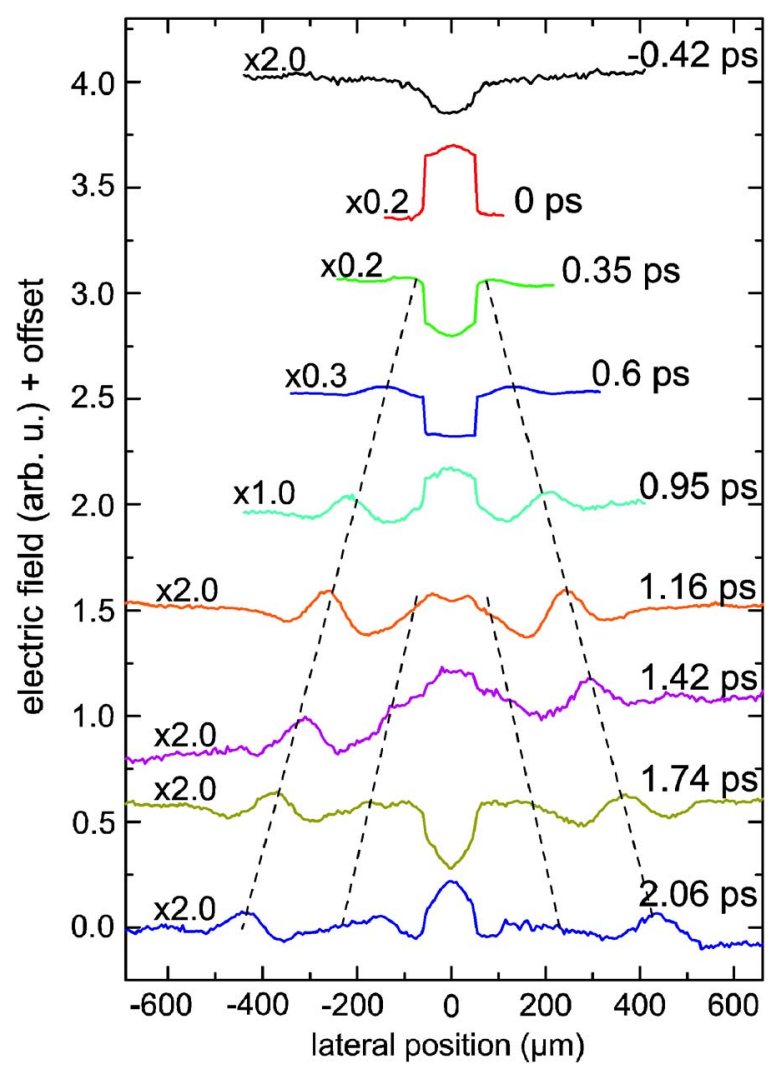

FIG. 3. (Color online) Lateral scans across the structure at consecutive time delays. The amplitudes are scaled differently as given in the figure. Dotted lines are guides to the eye and show positions of additional structures.

of the central negative peak, small positive peaks exist. With increasing time delay, they move further out to the left and to the right. The change in position of these additional peaks is indicated by the dotted lines. At about $\tau \approx 0.9 \mathrm{ps}$, two additional peaks start to appear next to the central peak. They are broader and smaller than the previous ones and exhibit an asymmetric shape.

The additional peaks appearing at positive time delays also depend on the exact structure of the investigated gold structure: when scanning two parallel $50 \mu \mathrm{m}$ wide gold stripes with $100 \mu \mathrm{m}$ distance (not shown), each of the extra structures in the changes in the terahertz signal at larger time delays consists of two peaks separated $\approx 100 \mu \mathrm{m}$ from each other.

The extra peaks can be explained by a resonance of the system sample-metallic tip. The system is excited by a broadband pulse with a spot size larger than the scanning range. Therefore the detected signal is not a simple interference pattern as observed in cw measurements. In a simplistic picture, a pulse travels back and forth between gold film and tip and is coupled out at the tip every round trip. The distances between the extra peaks and the gold structure in a line scan at time delay $\tau$ are approximately given by $d_{k}=c \tau /(2 k+1) n_{\text {eff }}$ (with $\left.k=0,1, \ldots\right)$, where $c$ is the velocity of light and $n_{\text {eff }}$ is the effective refractive index.

The positions $d_{0}$ of the outermost peaks versus time delay $\tau$ are shown in Fig. 4. A linear fit results in a slope of $(2.16 \pm 0.02) \times 10^{8} \mathrm{~m} / \mathrm{s}$ (assuming the shift in position is approximately the distance the pulse travels), resulting in $n_{\mathrm{eff}} \approx 1.39 \pm 0.01$. For comparison, the effective index is calculated for a half-space of the substrate according to $n_{\text {eff }}=\sqrt{\varepsilon_{\text {air }}+\varepsilon_{\text {substrate }}}$ using the refractive index of 1.95 for 


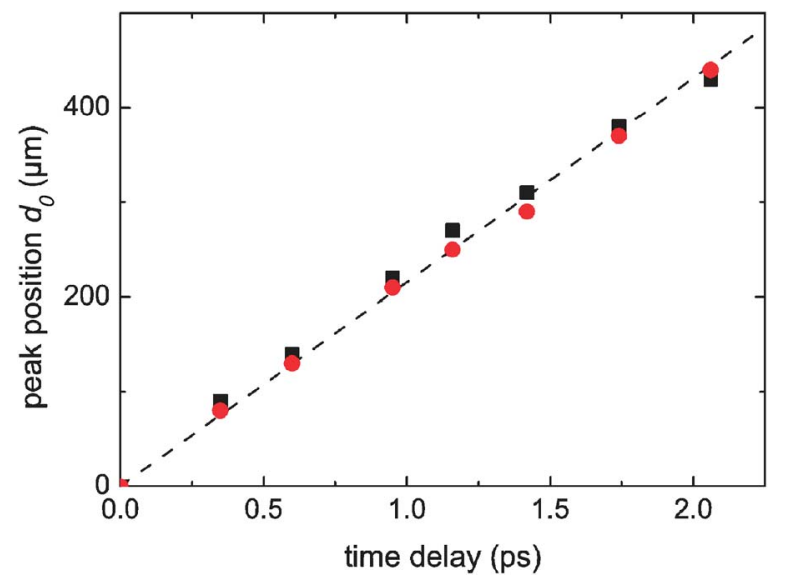

FIG. 4. (Color online) The position of the peaks (with $k=0$ ) outside the gold structure plotted vs the time delay. The dashed line is a linear fit to the average shift of the furthermost left (black squares) and right (red circles) peaks, yielding a velocity of $(2.16 \pm 0.02) \times 10^{8} \mathrm{~m} / \mathrm{s}$.

$\mathrm{SiO}_{2}$ in this wavelength range, ${ }^{27}$ which gives $n_{\text {eff }}=1.56$.

In addition, the position of the two peaks in the line scan at $\tau=2.06 \mathrm{ps}$ can be evaluated; the difference is $d_{0}-d_{1}$ $=275 \pm 20 \mu \mathrm{m}$. According to the above formula, this gives $n_{\text {eff }}=2 c \tau / 3\left(d_{0}-d_{1}\right)=1.5 \pm 0.1$ with a larger error due to the broadening of the inner peak $(k=1)$.

In conclusion, we observed the influence of the interaction of a tip with a metallic structure in a scanning terahertz near field microscope. This coupling leads to additional changes in the terahertz transient at distances much larger than the resolution limit of the microscope. The observed resonances between the tip and a metal structure could be of relevance when spectroscopic information is extracted from a terahertz scattering SNOM approach. The measurement of the full terahertz transient, which is necessary for performing a Fourier transform at each point of the scan, will be affected by contributions from the coupling to metallic structures of the sample further away.

The authors wish to thank M. Hagner for help with the sample preparation by electron beam lithography. Financial support through the SFB 513 and SFB 767 is acknowledged.
${ }^{1}$ G. C. Cho, P. Y. Han, X.-C. Zhang, and H. J. Bakker, Opt. Lett. 25, 1609 (2000).

${ }^{2}$ R. Huber, F. Tauser, A. Brodschelm, M. Bichler, G. Abstreiter, and A. Leitenstorfer, Nature (London) 414, 286 (2001).

${ }^{3}$ B. M. Fischer, M. Walther, and P. U. Jepsen, Phys. Med. Biol. 47, 3807 (2002).

${ }^{4}$ A. Markelz, IEEE J. Sel. Top. Quantum Electron. 14, 180 (2008).

${ }^{5}$ R. F. Soohoo, J. Appl. Phys. 33, 1276 (1962).

${ }^{6}$ E. A. Ash and G. Nicholls, Nature (London) 237, 510 (1972).

${ }^{7}$ D. W. Pohl, W. Denk, and M. Lanz, Appl. Phys. Lett. 44, 651 (1984).

${ }^{8}$ A. Lewis, M. Isaacson, A. Harootunian, and A. Muray, Ultramicroscopy 13, 227 (1984).

${ }^{9}$ G. A. Massey, J. A. Davis, S. M. Katnik, and E. Omon, Appl. Opt. 24, 1498 (1985).

${ }^{10}$ S. Hunsche, M. Koch, I. Brener, and M. Nuss, Opt. Commun. 150, 22 (1998).

${ }^{11}$ U. Schade, K. Holldack, P. Kuske, and G. Wüstefeld, Appl. Phys. Lett. 84, 1422 (2004).

${ }^{12}$ S. Mair, B. Gompf, and M. Dressel, Appl. Phys. Lett. 84, 1219 (2004).

${ }^{13}$ O. Mitrofanov, I. Brener, M. C. Wanke, R. R. Ruel, J. D. Wynn, A. J. Bruce, and J. Federic, Appl. Phys. Lett. 77, 591 (2000).

${ }^{14}$ J. F. Federici, O. Mitrofanov, M. Lee, J. Hsu, I. Brener, R. Harel, J. D. Wynn, L. N. Pfeiffer, and K. W. West, Phys. Med. Biol. 47, 3727 (2002).

${ }^{15}$ Q. Chen, Z. Jiang, G. X. Xu, and X.-C. Zhang, Opt. Lett. 25, 1122 (2000).

${ }^{16}$ F. Zenhausern, M. P. O’Boyle, and H. K. Wickramasinghe, Appl. Phys. Lett. 65, 1623 (1994).

${ }^{17}$ F. Keilmann, B. Knoll, and A. Kramer, Phys. Status Solidi B 215, 849 (1999).

${ }^{18}$ A. J. Adam, N. C. van der Valk, and P. C. Planken, J. Opt. Soc. Am. B 24, 1080 (2007).

${ }^{19}$ P. Planken, C. E. W. M. van Rijmenam, and R. Schouten, Semicond. Sci. Technol. 20, S121 (2005).

${ }^{20}$ K. Wang, D. M. Mittleman, N. van der Valk, and P. Planken, Appl. Phys. Lett. 85, 2715 (2004).

${ }^{21}$ R. Kersting, F. Buersgens, G. Acuna, and G. C. Cho, Adv. Solid State Phys. 47, 203 (2008).

${ }^{22}$ H. Zhan, V. Astley, M. Hvasta, J. A. Deibel, D. M. Mittleman, and Y.-S. Lim, Appl. Phys. Lett. 91, 162110 (2007).

${ }^{23}$ H.-G. von Ribbeck, M. Brehm, D. W. van der Weide, S. Winnerl, O. Drachenko, M. Helm, and F. Keilmann, Opt. Express 16, 3430 (2008).

${ }^{24}$ H.-T. Chen, R. Kersting, and G. C. Cho, Appl. Phys. Lett. 83, 3009 (2003).

${ }^{25}$ A. Dreyhaupt, S. Winnerl, M. Helm, and T. Dekorsy, Opt. Lett. 31, 1546 (2006).

${ }^{26}$ T. H. Chen, S. Kraatz, G. Cho, and R. Kersting, Phys. Rev. Lett. 93, 267401 (2004).

${ }^{27}$ E. D. Palik, Handbook of Optical Constants of Solids, Academic Press Handbook Series, edited by E. D. Palik (Academic, New York, 1985). 